www.nature.com/clinicalpractice/neph

even among those with known risk factors for CKD, including diabetes, hypertension and age $>60$ years. By contrast, tests for serum lipids and glucose were ordered for $71 \%$ and $33 \%$, respectively, of the total test population.

ICD-9-CM diagnostic codes for kidney disease were allocated by physicians in only $3 \%$ of cases. Code sensitivity for detecting estimated GFR $<60 \mathrm{ml} / \mathrm{min} / 1.73 \mathrm{~m}^{2}$ or $<30 \mathrm{ml} / \mathrm{min} / 1.73 \mathrm{~m}^{2}$ was $11 \%$ and $39 \%$, respectively, indicating that CKD is less likely to be detected in its early stages when intervention is most effective.

Guidelines recommending regular testing of high-risk patients for indicators of CKD seem to be ignored by many physicians. Opportunities for early intervention by nephrologists, and for implementation of preventative lifestyle initiatives by healthcare organizations, are therefore being lost. Furthermore, these findings call into question the accuracy of clinical studies of CKD cohorts identified from databases of diagnostic codes and laboratory data.

Rachael Williams

Original article Stevens LA et al. (2005) Low rates of testing and diagnostic codes usage in a commercial clinical laboratory: evidence for lack of physician awareness of chronic kidney disease. J Am Soc Nephrol 16: 2439-2448

\section{Contradictory guidelines for drug dose adjustment in renal impairment}

Secondary sources of drug information are remarkably inconsistent in their advice on adjusting dosages to account for impaired kidney function. This is the worrying conclusion of a systematic comparison of four widely used and hitherto well-respected publications: British National Formulary, Martindale: the Complete Drug Reference, Drug Prescribing in Renal Failure and American Hospital Formulary System Drug Information.

Vidal et al. found that each publication based its recommendations on a different definition of renal impairment, ranging from undefined qualitative terms to graded glomerular filtration rates. The rationale and search strategies underlying recommendations were not explained in detail. Most sources cited few or no primary references in support of their guidelines.

By comparing entries for the 100 drugs requiring dose-adjustment that were most commonly prescribed at Rabin Medical Center in 2003, including angiotensin-converting enzyme inhibitors, statins, antibiotics, corticosteroids, analgesics and diuretics, the authors also detected marked variation in the recommended dose modifications. For example, 11 drugs listed as requiring dose adjustment in one publication needed no modification according to another. Seven agents for which no adjustment was recommended in one source were categorized by a second as being contraindicated in patients with renal failure.

It seems that the standards of evidence-based practice are not being met by some producers of information upon which clinicians rely. A dearth of robust data might be contributing to the problem. Nevertheless, this analysis might spur those who compile drug-dosing guidelines to improve their data extraction methodologies, transparency of workflow, citation practices and consistency.

Rachael Williams

Original article Vidal L et al. (2005) Systematic comparison of four sources of drug information regarding adjustment of dose for renal function. BMJ 331: 263-266

\section{Choice of dialysis modality is affected by predialysis education}

End-stage renal disease can be treated using a range of renal replacement therapy (RRT) modalities. Uptake of the various modalities varies between countries and centers. This retrospective Belgian study reports that, in one center where patients were provided with predialysis education, most chose a self-care modality.

Of 242 patients beginning RRT during the 6-year study period, 57 were directed towards in-center hemodialysis, the majority for medical or psychosocial reasons. The rest were provided with information about RRT options, including continuous ambulatory peritoneal dialysis, automated peritoneal dialysis, in-center hemodialysis, self-care hemodialysis in a satellite unit and home hemodialysis. Education included individual discussion with a dialysis nurse in charge of the self-care modalities and viewing videos about the range of treatment options.

Eight patients underwent pre-emptive renal transplant. Of the remainder, $60 \%$ chose selfcare dialysis modalities. Patients choosing self care were, on average, younger than those choosing in-center modalities. Treatment choice

\section{GLOSSARY}

ICD-9-CM

International Classification of Disease, Ninth Revision, Clinical Modification 